\title{
Automatic Computing Methods for Special Functions
}

\author{
Irene A. Stegun and Ruth Zucker \\ Institute for Basic Standards, National Bureau of Standards, Washington, D.C. 20234
}

(May 25, 1970)

\begin{abstract}
Some of the pitfalls of automatic computation are described as well as their possible avoidance. A detailed description is given of the methods for computing the error and complementary error function. The implementation of the method is given in a double precision ASA FORTRAN program. The test ("driver") program and test results are also given.
\end{abstract}

Key words: Approximations; computer programs; computing pitfalls; continued fractions; error function; recurrence relations; series.

\section{Introduction}

AMS55[1] ${ }^{1}$ - the Handbook of Mathematical Functions -in addition to defining functions in terms of their integrals, power series, asymptotic expansions, etc., also supplies tables and approximating polynomials as well as methods of computing values both within and outside the tabular range. With the present trend to large scale computer systems, questions frequently arise concerning methods of computing the tabular values themselves. In many, if not most cases, the method used for computing the table is certainly not the best method for computing discrete values. While many specialized methods and programs exist $[3,6,7,8]$, as a supplement to the Handbook and to satisfy general needs, a systematic effort is being made to supply methods of computing special functions, accurate within machine precision, valid throughout the entire range of the function, efficient, and readily programmable and adaptable for varying precision and computers. Part I of the present paper is devoted to the error, probability, and related functions.

\section{General Methods}

Methods used in computing functions vary from expansion in series - power, Taylor, asymptotic, etc.; integration of the differential equation; numerical quadrature; evaluation of continued fractions; polynomial or rational approximations; recurrence techniques; iteration methods; stored tables with the use of interpolation; addition or duplication formulas, etc. Numerical quadrature and integration of the differential equation, while ideal for preparing a table, are generally inefficient for obtaining discrete values. Since the coefficients in approximating polynomials vary with precision, this method will be avoided if possible. The methods chosen for computing the functions will be those which avoid some of the common pitfalls or those pitfalls not easily remedied.

\section{Common Pitfalls and Their Avoidance}

Computation with alternating series leads to a loss of significant figures due to either or both subtraction and accumulated round-off errors. The use of Taylor series leads to difficulties of premature truncation if any of the derivatives possess zeros. The use of slowly convergent series leads to the difficulty of a heavy accumulation of round-off errors as well as of devising a satisfactory cut-off procedure. In computing with such series one might impose a condition on

' Figures in brackets indicate the literature references on p. 212. 
the number of terms to be used in the computation. However, this number varies with precision and the method would impose a timing penalty in certain ranges. After the $n$th term has satisfied the convergence criteria, the sum of the $n$th to the $(n+p)$ th term should be checked for its contribution. The loss of significant figures away from or at the zeros of the functions will be noted by comparing the largest term with the sum and the convergent term. This method of curtailing the series will lead to time savings and frequently avoid overflow and underflow conditions. In using asymptotic expansions the test for convergence should precede the test for divergence.

In computing with continued fractions [2] to avoid premature truncation and or possible overflow or underflow difficulties, the convergents should only be obtained after the numerator and denominator of the ratio are tested for zeros. In this circumstance, the test for convergence should likewise be omitted. In continued fractions where the partial numerators and denominators are always positive, no zeros arise and the value of the continued fraction always lies between two consecutive convergents, except possibly for round-off errors in the calculation. Use of the transformed continued fraction containing only the even or odd convergents of the original fraction, while more efficient generally, may lead in certain cases to a loss of significant figures and to overflow conditions more quickly.

In recurrence techniques, there may be a loss of significant figures or a heavy buildup of round-off errors. Forward and backward techniques or a combination of both may be useful [4, 5]. Attention must be paid to the proper choice of normalization factor, avoiding the use of values at or near zeros.

In using iterative methods, common pitfalls are the imposition of too stringent a convergence criteria and/or a failure to impose a limit on the maximum number of iterations.

\section{General Procedures}

In addition to detailing the methods of computing, the ASA FORTRAN programs will be presented, prepared in such a way that only a minimal number of changes need be made to accommodate the change in precision. Initially the test ("driver") program will also be given as well as test results and timing in the hope of detecting software and hardware errors.

The authors, hoping to satisfy the needs of all users, earnestly solicit suggestions for improvements and comments relative to testing on other computers. In this way it is hoped that an excellent library of special functions will be established.

\section{References}

[1] Abramowitz, M., and Stegun, I. A., Handbook of Mathematical Functions, Nat. Bur. Stand. (U.S.), Applied Mathematics Series 55 (U. S. Government Printing Office, Washington, D. C., 1964).

[2] Blanch, G., Numerical evaluation of continued fractions, SIAM Review 6, 4, 383-421 (1964).

[3] Communications of the ACM (Association for Computing Machinery), Collected Algorithms, New York (1960- . )

[4] Gautschi, W., Recursive computation of special functions, U. Mich. Engineering Summer Conference, Numerical Analysis, Ann Arbor (1963).

[5] Miller, J. C. P., and Brown, D. J. Spencer, An algorithm for evaluation of remote terms in a linear recurrence sequence, Comp. J. 9, 2, 188-190 (1966).

[6] Nordisk Tidskrift For Informations-Behandling (BIT), Copenhagen (1961- ).

[7] Numerische Mathematik, Handbook Series Special Functions (Springer-Verlag, Berlin, 1963-

[8] The Computer Journal, Algorithm Supplement, The British Computer Society, London (1964-

[9] Wall, H. S., Analytic Theory of Continued Fractions (D. Van Nostrand Co., New York, 1948). 


\section{Automatic Computing Methods for Special Functions}

Part I. Error, Probability, and Related Functions

\section{Definitions}

Error Function

$$
\begin{aligned}
& \operatorname{erf} x=\frac{2}{\sqrt{\pi}} \int_{0}^{x} e^{-t^{2}} d t \\
& \operatorname{erfc} x=\frac{2}{\sqrt{\pi}} \int_{x}^{\infty} e^{-t^{2}} d t \\
& =1-\operatorname{erf} x \\
& \operatorname{erf}(-x)=-\operatorname{erf} x \\
& \operatorname{erfc}(-x)=2-\operatorname{erfc} x \\
& Z(x)=\frac{1}{\sqrt{2 \pi}} e^{-x^{2} / 2} \quad A(x)=\frac{1}{\sqrt{2 \pi}} \int_{-x}^{x} e^{-t^{2} / 2} d t=\int_{-x}^{x} Z(t) d t \\
& =\operatorname{erf}(x / \sqrt{2}) \\
& P(x)=\frac{1}{\sqrt{2 \pi}} \int_{-\infty}^{x} e^{-t^{2} / 2} d t \quad Q(x)=\frac{1}{\sqrt{2 \pi}} \int_{x}^{\infty} e^{-t^{2} / 2} d t \\
& =\int_{-\infty}^{x} Z(t) d t \quad=\int_{x}^{\infty} Z(t) d t \\
& =\frac{1}{2}[1+\operatorname{erf}(x / \sqrt{2})] \quad=1-P(x)=\frac{1}{2} \operatorname{erfc}(x / \sqrt{2})
\end{aligned}
$$

Confluent Hypergeometric Function.

$$
\begin{gathered}
M\left(\frac{1}{2}, \frac{3}{2},-x^{2}\right)=(\sqrt{\pi} / 2 x) \text { erf } x \\
M\left(1, \frac{3}{2}, x^{2}\right)=(\sqrt{\pi} / 2 x) e^{x^{2}} \text { erf } x \\
U\left(\frac{1}{2}, \frac{1}{2}, x^{2}\right)=\sqrt{\pi} e^{x^{2}} \operatorname{erfc} x
\end{gathered}
$$

Incomplete Gamma Function

$$
\begin{aligned}
& \gamma\left(\frac{1}{2}, x^{2}\right)=2 \int_{0}^{x} e^{-t^{2}} d t=\sqrt{\pi} \operatorname{erf} x \\
& \Gamma\left(\frac{1}{2}, x^{2}\right)=2 \int_{x}^{\infty} e^{-t^{2}} d t=\sqrt{\pi} \operatorname{erfc} x
\end{aligned}
$$

\section{Method}

$x$, a real variable

$\mathrm{NBC}=$ the number of binary digits in the characteristic of a floating point number

$\mathrm{NBM}=$ accuracy desired or the maximum number of binary digits in the mantissa of a floating point number

For $x=0$, erf $x=0$; erfc $x=1.0$. 
The power series is used for $|x| \leqslant \operatorname{ULPS}(=1)$, the upper limit for the power series

$$
\operatorname{erf} x=\frac{2 x}{\sqrt{\pi}} e^{-x^{2}} \sum_{N=0}^{\infty} \frac{\left(2 x^{2}\right)^{N}}{1 \cdot 3 \ldots(2 N+1)}=\frac{2 x}{\sqrt{\pi}} e^{-x^{2}}\left[\sum_{N=1}^{M} T_{N}+1\right]
$$

where $T_{0}=1, D_{0}=1, T_{N}=\frac{2 x^{2}}{2 N+1} T_{N-1}=\frac{\mathrm{PWR}}{D_{N}} T_{N-1} ; D_{N}=D_{N-1}+2 ; N \geqslant 1$.

Since $\min \sum_{0}^{\infty} T_{N}=1$, it is valid to obtain $M$ for the final term by testing for

$$
T_{M} \leqslant \text { TOLER }\left(=2^{-\mathrm{NBM}}\right)
$$

Note: In the program, in order to minimize round-off errors, summing starts at $N=1$.

One then obtains erfc $x=1-\operatorname{erf} x, \operatorname{erf}(-x)=-\operatorname{erf} x$, erfc $(-x)=2-\operatorname{erfc} x$.

The continued fraction

$$
\operatorname{erfc} x=\frac{e^{-x^{2}}}{\sqrt{\pi}}\left[\frac{2 x}{2 x^{2}+1}-\frac{1 \cdot 2}{2 x^{2}+5}-\frac{3 \cdot 4}{2 x^{2}+9}-\ldots\right]=\frac{e^{-x^{2}}}{\sqrt{\pi}} F
$$

is used for $1<|x| \leqslant \mathrm{ULCF}\left(=0.83\left[2^{(\mathrm{NBC}-1) / 2}\right]\right)$, the upper limit (machine bound) for the continued fraction. The above continued fraction yields only even convergents, forming a monotonically increasing sequence, of the following continued fraction

$$
\operatorname{erfc} x=\frac{e^{-x^{2}}}{\sqrt{\pi}}\left[\frac{1}{x}+\frac{1 / 2}{x+} \frac{1}{x}+\frac{3 / 2}{x+} . . .\right] \text {. }
$$

Setting

$F_{-1}=0, F_{0}=2 x, G_{-1}=1, G_{0}=2 x^{2}+1, W_{1}=1, B_{1}=G_{0}+4$ with $W_{N}=W_{N-1}+2, B_{N}=B_{N-1}+4, N>1$, one obtains

$$
A_{N}=-W_{N}\left(W_{N}+1\right), F_{N}=A_{N} F_{N-2}+B_{N} F_{N-1}, G_{N}=A_{N} G_{N-2}+B_{N} G_{N-1}, N \geqslant 1 .
$$

If $\left|1-\frac{F_{N} / G_{N}}{F_{N-1} / G_{N-1}}\right| \leqslant \operatorname{TOLER}\left(=2^{-\mathrm{NBM}}\right)$, then $F_{N} / G_{N}=F$. If $F_{N-1} / G_{N-1} \operatorname{denoted}$ by $\mathrm{PREV} \leqslant F_{N} / G_{N}$ the sequence is permitted to continue after first checking for boundedness $\left(G_{N}<\right.$ scaling factor, $\mathrm{SCF}=2^{C 2-\mathrm{NBC}}$ where $\left.C 2=2^{\mathrm{NBC}-1}\right)$. If $\mathrm{PREV}>F_{N} / G_{N}, \mathrm{PREV}=F$. One then obtains the quantities

$$
\operatorname{erf} x=1-\operatorname{erfc} x, \operatorname{erf}(-x)=-\operatorname{erf} x, \operatorname{erfc}(-x)=2-\operatorname{erfc} x \text {. }
$$

For $|x|>0.83\left[2^{(\mathrm{NBC}-1) / 2}\right]$, erf $|x|=1$, erfc $|x|=0$, the limiting values of the functions.

Beyond $|x|=1$, a loss of significant figures will occur in subtraction in obtaining erfc $x$, and in addition round-off errors begin to accumulate more rapidly. If only erf $x$ is needed, a time saving is achieved by extending the power series to $|x|=2$. The continued fraction should then be used until the appropriate critical value is reached.

While the continued fraction is convergent for $x>0$, the number of convergents needed increases rapidly and without scaling underflow or overflow occur for $x$ nearing zero. For $\mathrm{NBC}=8$, $\mathrm{NBM}=27$, underflow occurred near $x=0.91$. The partial numerators and denominators $F_{N-1}$, 
$G_{N-1}, F_{N-2}$ and $G_{N-2}$ are scaled but greater time saving is achieved by using the power series. If one uses the continued fraction for $|x|<0.61$ both $F_{N}$ and $G_{N}$ must be tested for boundedness.

The maximum precision attainable with the use of the asymptotic expansion

$$
\operatorname{erfc} x=\frac{e^{-x^{2}}}{\sqrt{\pi} x}\left(1+\sum_{N=1}^{\infty} \frac{(-1)^{N} 1 \cdot 3 \ldots(2 N-1)}{\left(2 x^{2}\right)^{N}}\right)
$$

is for

$$
\begin{aligned}
& x=4.6,|\epsilon| \leqslant 1 \text { unit in the 9th significant figure, } \\
& x=6.7,|\epsilon| \leqslant 1 \text { unit in the 19th significant figure. }
\end{aligned}
$$

\begin{tabular}{|c|c|c|}
\hline & \multicolumn{2}{|c|}{ Maximum number of terms } \\
\hline & $\mathrm{NBM}=27$ & $\mathrm{NBM}=60$ \\
\hline Power series $-\operatorname{erf} 1 \ldots \ldots \ldots \ldots \ldots \ldots \ldots \ldots \ldots \ldots \ldots \ldots \ldots \ldots \ldots$ & 13 & 21 \\
\hline Continued fraction $-\operatorname{erfe} 1 \ldots \ldots \ldots \ldots \ldots \ldots$ & 24 & 124 \\
\hline Asymptotic expansion-erfc $6.7 \ldots \ldots \ldots \ldots \ldots$ & 9 & 47 \\
\hline erfe $9.3 \ldots \ldots \ldots \ldots \ldots$ & 6 & 17 \\
\hline Continued fraction - erfe $6.7 \ldots \ldots \ldots \ldots \ldots \ldots$ & 3 & 8 \\
\hline erfe $9.3 \ldots \ldots \ldots \ldots \ldots \ldots$ & 3 & 6 \\
\hline
\end{tabular}

The following table gives an indication of the number of terms needed for particular values of NBM

\section{Range}

The range covered is limited by the machine bound for the exponential function.

For $|x| \leqslant 0.83\left[2^{(\mathrm{NBC}-1) / 2}\right]$, the computations are performed with $|x| \leqslant 9.3$ for $\mathrm{NBC}=8,|x| \leqslant 26.5$ for $\mathrm{NBC}=11$.

For $|x|>.83\left[2^{(\mathrm{NBC}-1) / 2}\right]$, erf $|x|=1$, erfc $|x|=0$.

\section{Accuracy}

At least (NBM-I-3) significant binary digits where $\mathrm{I}$ is the number of binary digits representing the integer part of $x^{2}$. (This is essentially the accuracy of the exponential routine, with round-off error allowance.)

\section{Precision}

\begin{tabular}{|c|c|c|c|c|}
\hline For the range & & $\mathrm{NBM}=27$ & $\mathrm{NBM}=60$ & Method \\
\hline $\begin{array}{l}x=0(.005) 1 \ldots \ldots \ldots . \\
x=1(.05) 9.4 \ldots \ldots \ldots . \\
x=9.2(.2) 26.6 \ldots \ldots\end{array}$ & $\begin{array}{l}(201 \text { values }) \ldots \ldots \ldots \ldots \ldots \\
(169 \text { values }) \ldots \ldots \ldots \ldots \ldots \ldots \\
(88 \text { values }) \ldots \ldots \ldots \ldots \ldots \ldots\end{array}$ & $\begin{array}{r}0.08 \\
.16 \\
\cdots\end{array}$ & $\left.\begin{array}{r}0.22 \\
.53 \\
.13\end{array}\right\}$ & \multirow[t]{2}{*}{$\begin{array}{l}\text { Power series } \\
\text { Continued } \\
\text { fraction }\end{array}$} \\
\hline \multicolumn{2}{|c|}{ Maximum Time/Evaluation (Seconds).......... } & .003 & .015 & \\
\hline
\end{tabular}

Variable-by setting the desired NBM.

6. TIMING (Seconds-Univac 1 108) 


\section{Testing}

To avoid errors due to incorrect arguments, the beginning argument, the interval, and end argument were read in as scaled integers. The generation was performed with integer values, double precision conversion made, then single precision values obtained.

The double precision results were checked, when possible, against those obtained by using the polynomial or rational approximations of Clenshaw [1], ${ }^{1}$ Cody [2], Hart [3], and Strecok [6]. In addition, special values were checked by the asymptotic expansion and numerical integration. The single precision results were then checked against the double precision results. In all cases the results obtained agreed within the reported accuracy.

For those desiring both functions accurately with limited precision throughout the range, the approximations of Cody were found the most efficient and accurate.

\section{Critical Values}

$$
\begin{aligned}
& \text { erfe } 9.3=0.1653244184030157(-38)(\mathrm{NBC}=8) \\
& \text { erfe } 26.5=0.221090766426373(-306)(\mathrm{NBC}=11) \\
& \operatorname{erf} 4.33 \\
& |\epsilon|<1(-9) \quad \mathrm{NBM}=27 \\
& \text { erf } 4.82 \\
& =1-\epsilon \\
& |\epsilon|<1(-11) \mathrm{NBM}=36 \\
& \text { erf } 5.68 \\
& |\epsilon|<1(-15) \mathrm{NBM}=48 \\
& \text { erf } 6.43 \\
& |\epsilon|<1(-19) \mathrm{NBM}=60
\end{aligned}
$$

\section{Special Constants}

$$
\begin{array}{rllllll}
2(\pi)^{-\frac{1}{2}}=1.12837 & 91670 & 95512 & 57389 & 61589 & 03121 \\
(\pi)^{\frac{1}{2}} / 2=0.88622 & 69254 & 52758 & 01364 & 90837 & 41670 \\
(\pi)^{-\frac{1}{2}}=0.56418 & 95835 & 47756 & 28694 & 80794 & 51560 \\
\pi^{\frac{1}{2}}=1.77245 & 38509 & 05516 & 02729 & 81674 & 83341 \\
(2 \pi)^{-\frac{1}{2}}=0.39894 & 22804 & 01432 & 67793 & 99460 & 59934 \\
(2 \pi)^{\frac{1}{2}}=2.50662 & 82746 & 31000 & 50241 & 57652 & 84811 \\
\pi & =3.14159 & 26535 & 89793 & 23846 & 26433 & 83279 \\
(2)^{-\frac{1}{2}}=0.70710 & 67811 & 86547 & 52440 & 08443 & 62104 \\
2^{\frac{1}{2}}=1.41421 & 35623 & 73095 & 04880 & 16887 & 24209
\end{array}
$$

$$
\begin{aligned}
& \text { erf } 1=0.84270 \quad 07929 \quad 49714 \quad 86934 \quad 12206 \\
& \text { erf } \rho=0.5 \\
& \rho=0.47693 \quad 62762 \quad 04469 \quad 87338
\end{aligned}
$$

${ }^{1}$ Figures in brackets indicate the literature references on p. 217. 


$$
\begin{array}{lllllllllll}
2^{-27}=0.74505 & 80596 & 92382 & 8125 & (-8) & & & & \\
2^{-36}=0.14551 & 91522 & 83668 & 51806 & 64062 & 5 & (-10) & & & \\
2^{-48}=0.35527 & 13678 & 80050 & 09293 & 55621 & 33789 & 0625 & (-14) & & \\
2^{-60}=0.86736 & 17379 & 88403 & 54720 & 59622 & 40695 & 95336 & 91406 & 25 & (-18)
\end{array}
$$

\section{References}

[1] Clenshaw, C. W., Chebyshev Series for Mathematical Functions, National Physical Laboratory Mathematical Tables, 5, London, 1962.

[2] Cody, W. J., Rational Chebyshev approximations for the error function, Math. of Comp. 23, 631-637 (1969).

[3] Hart, John F. et al., Computer Approximations, The SIAM Series in Applied Mathematics (John Wiley \& Sons, Inc., New York, 1968).

[4] National Bureau of Standards, Tables of Normal Probability Functions, Applied Mathematics Series 23 (U.S. Government Printing Office, W ashington, D.C., 1953).

[5] National Bureau of Standards, Tables of the Error Function and its Derivative, Applied Mathematics Series 41 (U.S. Government Printing Office, Washington, D.C., 1954).

[6] Strecol, Anthony J., On the calculation of the inverse of the error function, Math. of Comp. 22, 144-158 (1968). 


\begin{tabular}{|c|c|c|}
\hline $1^{*}$ & & SUBROUTINE ERRINT (X, ERF, ERFC) \\
\hline $2^{*}$ & $\mathrm{C}$ & (ASA FORTRAN) \\
\hline $3^{*}$ & $\mathrm{C}$ & CALL ERRINT ( $\mathrm{X}, \mathrm{ERF}, \mathrm{ERFC})$ \\
\hline $4^{*}$ & $\mathrm{C}$ & ENTERING WITH X(A REAL VARIABLE), ONE OBTAINS BOTH ERF X \\
\hline $5^{*}$ & $\mathrm{C}$ & AND ERFC X TO THE MAXIMUM MACHINE ACCURACY POSSIBLE. \\
\hline $6^{*}$ & $\mathrm{C}$ & \\
\hline $7 *$ & $\mathrm{C}$ & THE PROGRAM, AS SET UP BELOW \\
\hline $8^{*}$ & $\mathrm{C}$ & ( 1 ) WITH THE DOUBLE PRECISION TYPE STATEMENT, \\
\hline $9 *$ & $\mathrm{C}$ & (2) $\mathrm{NBC}=11, \mathrm{NBM}=60$ ON THE FIRST DATA CARD AND \\
\hline $10^{*}$ & $\mathrm{C}$ & (3) THE REMAINING CONSTANTS ON THE SECOND AND THIRD DATA \\
\hline $11^{*}$ & $\mathrm{C}$ & CARDS GIVEN AS DOUBLE PRECISION CONSTANTS, YIELDS DOUBLE \\
\hline $12^{*}$ & $\mathrm{C}$ & PRECISION RESULTS ON THE UNIVAC 1108 SYSTEM. \\
\hline $13^{*}$ & $\mathrm{C}$ & \\
\hline $14^{*}$ & $\mathrm{C}$ & SINGLE PRECISION RESULTS ARE OBTAINED BY \\
\hline $15^{*}$ & $\mathrm{C}$ & ( 1 ) DELETING THE TYPE STATEMENT \\
\hline $16^{*}$ & $\mathrm{C}$ & (2) SETTING NBC=8, NBM=27 ON THE FIRST DATA CARD \\
\hline $17 *$ & $\mathrm{C}$ & (3) CHANGING THE D 'S TO E 'S ON THE SECOND AND THIRD DATA \\
\hline $18^{*}$ & $\mathrm{C}$ & CARDS. \\
\hline $19 *$ & $\mathrm{C}$ & (4) CHANGING FUNCTION TYPE-DABS TO ABS, DEXP TO EXP \\
\hline $20 *$ & $\mathrm{C}$ & FOR OTHER VALUES OF NBM, THE CONSTANT ON THE THIRD DATA \\
\hline $21 *$ & $\mathrm{C}$ & CARD SHOULD BE GIVEN TO THE CORRESPONDING PRECISION. \\
\hline $22^{*}$ & $\mathrm{C}$ & \\
\hline $23^{*}$ & $\mathrm{C}$ & THE SUBROUTINE CANNOT READILY BE ADAPTED TO \\
\hline $24^{*}$ & $\mathrm{C}$ & COMPUTE THE ERROR FUNCTION FOR A COMPLEX \\
\hline $25^{*}$ & $\mathrm{C}$ & ARGUMENT \\
\hline $26^{*}$ & $\mathrm{C}$ & $\operatorname{ERF}(X)=(2 / \mathrm{SQRT}(\mathrm{PI})) *$ INTEGRAL FROM $0 \mathrm{TO} \mathrm{X}$ OF EXP $\left(-\mathrm{T}^{*}{ }^{*} 2\right) \mathrm{DT}$ \\
\hline $27 *$ & $\mathrm{C}$ & $\operatorname{ERFC}(X)=(2 /$ SQRT $(P I)) *$ INTEGRAL FROM X TO INFINITY OF \\
\hline $28 *$ & $\mathrm{C}$ & $\operatorname{EXP}\left(-\mathrm{T}^{* *} 2\right) \mathrm{DT}=1-\operatorname{ERF}(\mathrm{X})$ \\
\hline $29 *$ & $\mathrm{C}$ & \\
\hline $30 *$ & $\mathrm{C}$ & $\operatorname{ERF}(-X)=-\operatorname{ERF}(X)$ \\
\hline $31 *$ & $\mathrm{C}$ & $\operatorname{ERFC}(-X)=2-\operatorname{ERFC}(X)$ \\
\hline $32^{*}$ & $\mathrm{C}$ & \\
\hline $33^{*}$ & $\mathrm{C}$ & NBC=NO. OF BINARY DIGITS IN THE CHARACTERISTIC OF A FLOATING POINT \\
\hline $34^{*}$ & $\mathrm{C}$ & NUMBER \\
\hline $35^{*}$ & $\mathrm{C}$ & NBM=ACCURACY DESIRED OR MAX. NO. OF BINARY DIGITS IN THE MANTISSA OF \\
\hline $36 *$ & $\mathrm{C}$ & A FLOATING POINT NUMBER. \\
\hline $37 *$ & $\mathrm{C}$ & \\
\hline $38 *$ & $\mathrm{C}$ & POWER SERIES FOR ABS $(X)$.LE. 1 ,ULPS, UPPER LIMIT FOR \\
\hline $39 *$ & $\mathrm{C}$ & SERIES, CONTINUED FRACTION FOR ABS (X) . GT . I AND . LE. \\
\hline $40 *$ & $\mathrm{C}$ & ULCF (UPPER LIMIT OF CONTINUED FRACTION) \\
\hline 41 * & $\mathrm{C}$ & \\
\hline $42^{*}$ & $\mathrm{C}$ & $\operatorname{ABS}(\mathrm{X}) \cdot \mathrm{LE} \cdot \mathrm{ULCF} \quad \mathrm{ULCF}=.83^{*}\left(2 \cdot{ }^{* *}((\mathrm{NBC}-1) / 2)\right)$ \\
\hline $43^{*}$ & $\mathrm{C}$ & ABS (ULCF ) APPROX. $9.3, \mathrm{NBC}=8$ \\
\hline $44^{*}$ & $\mathrm{C}$ & $26.5, \mathrm{NBC}=11$ \\
\hline $45^{*}$ & $\mathrm{C}$ & BEYOND THIS RANGE THE LIMITING VALUES OF THE FUNCTIONS \\
\hline $46^{*}$ & $\mathrm{C}$ & ARE SUPPLIED $\quad \operatorname{ERF}($ INF $)=1.0, \quad \operatorname{ERFC}(I N F)=0$ \\
\hline $47 *$ & $\mathrm{C}$ & \\
\hline $48 *$ & $\mathrm{C}$ & ROUTINE WILL RETURN (NBM-I-3) SIGNIFICANT BINARY DIGITS \\
\hline $49 *$ & $\mathrm{C}$ & WHERE I IS THE NUMBER OF BINARY DIGITS REPRESENTING THE \\
\hline
\end{tabular}




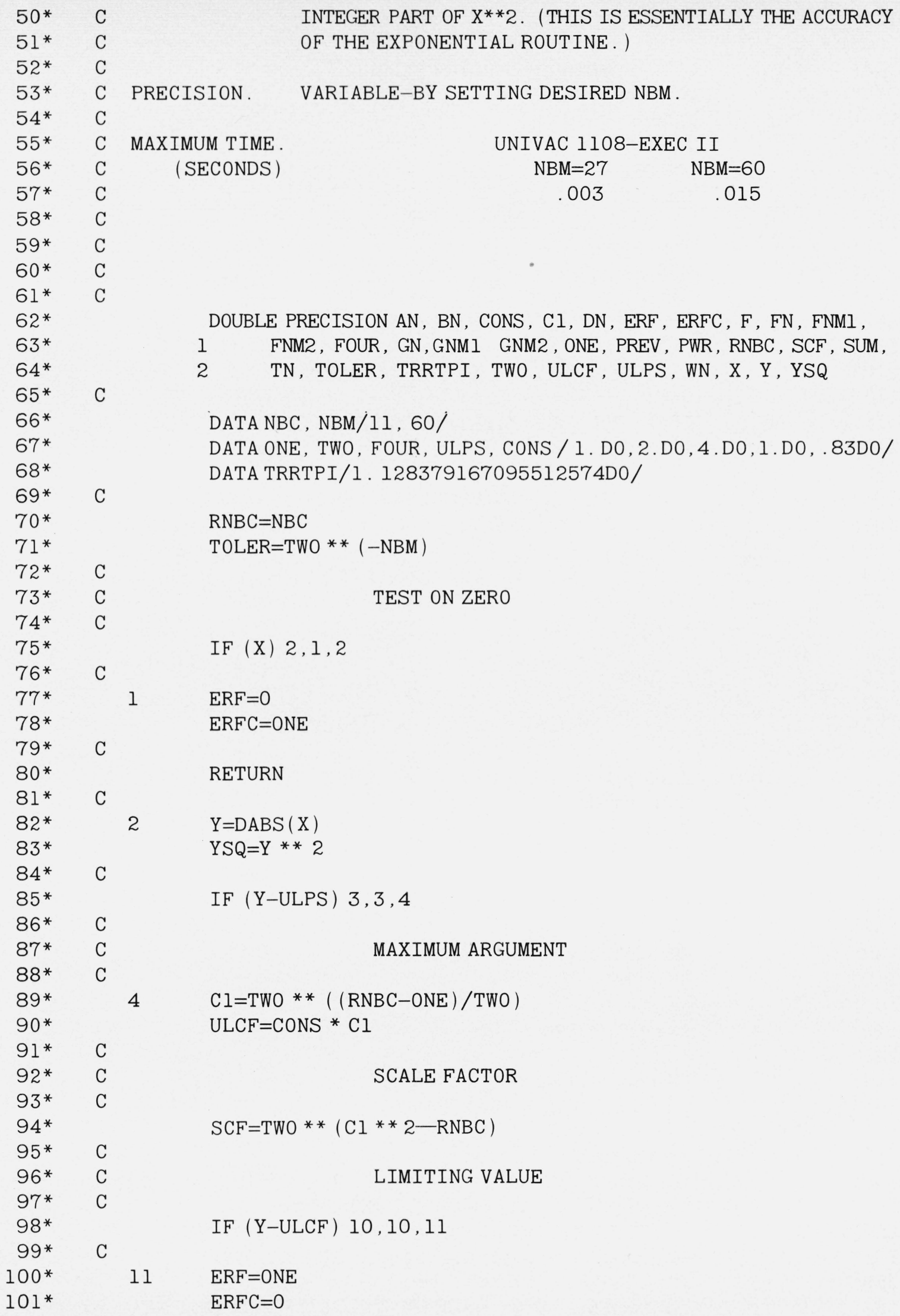




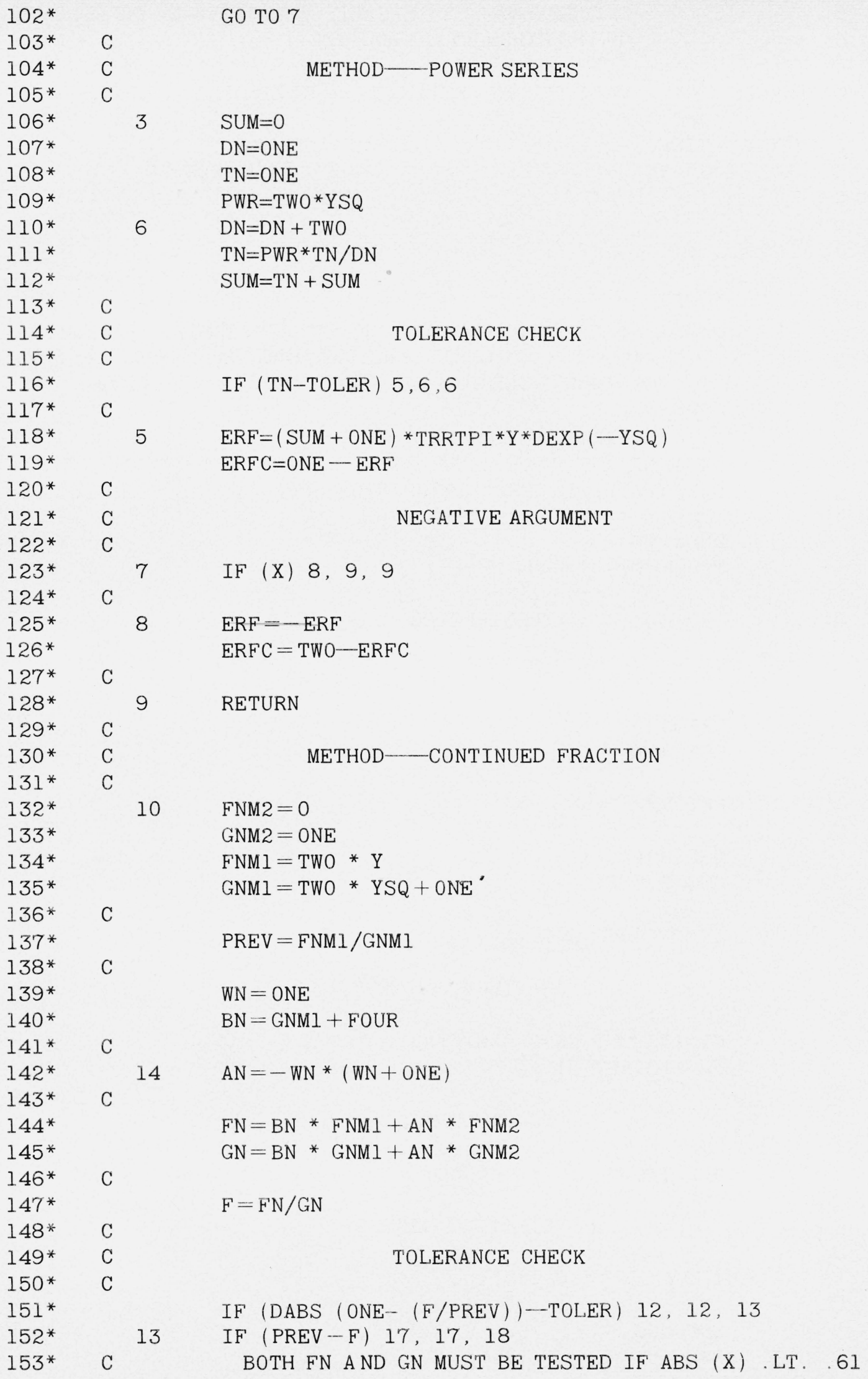

(n) 


\begin{tabular}{|c|c|c|}
\hline $154^{*}$ & 17 & IF (GN . LT. SCF) GO TO 16 \\
\hline $155^{*}$ & $\mathrm{C}$ & \\
\hline $156^{*}$ & $\mathrm{C}$ & SCALING \\
\hline $157^{*}$ & $\mathrm{C}$ & \\
\hline $158^{*}$ & 15 & $F N=F N / S C F$ \\
\hline $159 *$ & & $\mathrm{GN}=\mathrm{GN} / \mathrm{SCF}$ \\
\hline $160 *$ & & $\mathrm{FNMI}=\mathrm{FNMI} / \mathrm{SCF}$ \\
\hline $161^{*}$ & & $\mathrm{GNMI}=\mathrm{GNMI} / \mathrm{SCF}$ \\
\hline $162^{*}$ & C & \\
\hline $163 *$ & 16 & FNM $2=$ FNMI \\
\hline $164^{*}$ & & GNM2 = GNM1 \\
\hline $165 *$ & & $\mathrm{FNMI}=\mathrm{FN}$ \\
\hline $166^{*}$ & & $\mathrm{GNMI}=\mathrm{GN}$ \\
\hline $167 *$ & $\mathrm{C}$ & \\
\hline $168^{*}$ & & $\mathrm{WN}=\mathrm{WN}+\mathrm{TWO}$ \\
\hline $169 *$ & & $\mathrm{BN}=\mathrm{BN}+\mathrm{FOUR}$ \\
\hline $170 *$ & & $\mathrm{PREV}=\mathrm{F}$ \\
\hline $171^{*}$ & & G0 T0 14 \\
\hline $172^{*}$ & $\mathrm{C}$ & \\
\hline $173^{*}$ & 18 & $F=P R E V$ \\
\hline $174^{*}$ & 12 & $\mathrm{ERFC}=\mathrm{F}^{*} \mathrm{DEXP} \quad(-\mathrm{YSQ})^{*} \mathrm{TRRTPI} / \mathrm{TWO}$ \\
\hline $175 *$ & & $\mathrm{ERF}=\mathrm{ONE}-\mathrm{ERFC}$ \\
\hline $176^{*}$ & $\mathrm{C}$ & \\
\hline $177^{*}$ & & G0 T0 7 \\
\hline $178 *$ & $\mathrm{C}$ & \\
\hline $179 *$ & & END \\
\hline
\end{tabular}




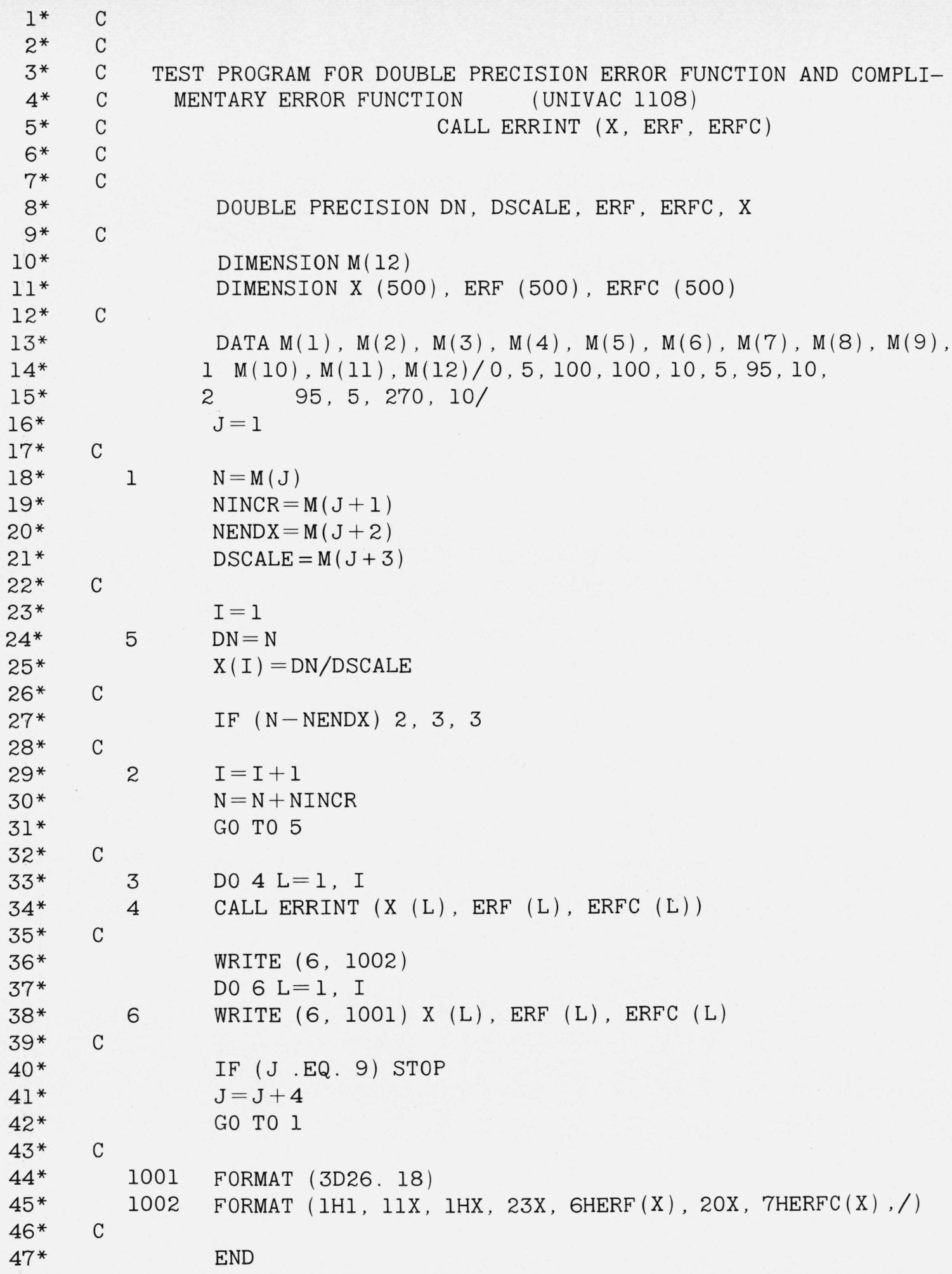


.000000000000000000

$.500000000000000000-001$

$.100000000000000000+000$

$.150000000000000000+000$

$.200000000000000000+000$

$.250000000000000000+000$

$.300000000000000000+000$

$.350000000000000000+000$

$.400000000000000000+000$

$.450000000000000000+000$

$.500000000000000000+000$

$.549999999999999999+000$

$.599999999999999999+000$

$.650000000000000000+000$

$.700000000000000000+000$

$.750000000000000000+000$

$.799999999999999999+000$

$.849999999999999999+000$

$.900000000000000000+000$

$.950000000000000000+000$

$.100000000000000000+001$

\section{$x$}

$.100000000000000000+001$ $.150000000000000000+001$ $.200000000000000000+001$ $.250000000000000000+001$ $.300000000000000000+001$ $.350000000000000000+001$ $.400000000000000000+001$ $.450000000000000000+001$ $.500000000000000000+001$ $.549999999999999999+001$ $.599999999999999999+001$ $.650000000000000000+001$ $.700000000000000000+001$ $.750000000000000000+001$ $.799999999999999999+001$ $.849999999999999999+001$ $.900000000000000000+001$ $.950000000000000000+001$
.000000000000000000

$.563719777970166236-001$

$.112462916018284892+000$

$.167995971427363495+000$

$.222702589210478454+000$

$.276326390168236935+000$

$.328626759459127429+000$

$.379382053562310322+000$

$.428392355046668455+000$

$.475481719786923677+000$

$.520499877813046541+000$

$.563323366325108957+000$

$.603856090847925924+000$

$.642029327355671843+000$

$.677801193837418476+000$

$.711155633653515133+000$

$.742100964707660485+000$

$.770668057608352530+000$

$.796908212422832131+000$

$.820890807273277944+000$

$.842700792949714871+000$

$\operatorname{ERF}(X)$

$.842700792949714871+000$
$.966105146475310728+000$
$.995322265018952735+000$
$.999593047982555043+000$
$.999977909503001415+000$
$.999999256901627659+000$
$.999999984582742101+000$
$.999999999803383957+000$
$.999999999998462541+000$
$.999999999999992643+000$
$.999999999999999979+000$
$.100000000000000000+001$
$.100000000000000000+001$
$.100000000000000000+001$
$.100000000000000000+001$
$.100000000000000000+001$
$.100000000000000000+001$
$.100000000000000000+001$
$.100000000000000000+001$ $.943628022202983377+000$ $.887537083981715109+000$ $.832004028572636506+000$ $.777297410789521546+000$ $.723673609831763065+000$ $.671373240540872572+000$ $.620617946437689678+000$ $.571607644953331545+000$ $.524518280213076324+000$ $.479500122186953459+000$ $.436676633674891044+000$ $.396143909152074076+000$ $.357970672644328157+000$ $.322198806162581524+000$ $.288844366346484867+000$ $.257899035292339516+000$ $.229331942391647470+000$ $.203091787577167870+000$ $.179109192726722056+000$ $.157299207050285130+000$

\section{$\operatorname{ERFC}(X)$}

$.157299207050285130+000$ $.338948535246892735-001$ $.467773498104726582-002$ $.406952017444958938-003$ $.220904969985854420-004$ $.743098372341412774-006$ $.154172579002800195-007$ $.196616044154288756-009$ $.153745979442803493-011$ $.735784791797439867-014$ $.215197367124989151-016$ $.384214832712064783-019$ $.418382560777941505-022$ $.277664938603056939-025$ $.112242971729829286-028$ $.276232407133377193-032$ $.413703174651381081-036$ $.376921448565488099-040$ 


$\begin{array}{ll}.950000000000000000+001 & .100000000000000000+001 \\ .100000000000000000+002 & .100000000000000000+001 \\ .10500000000000000+002 & .100000000000000000+001 \\ .110000000000000000+002 & .100000000000000000+001 \\ .115000000000000000+002 & .100000000000000000+001 \\ .120000000000000000+002 & .100000000000000000+001 \\ .125000000000000000+002 & .100000000000000000+001 \\ .130000000000000000+002 & .100000000000000000+001 \\ .135000000000000000+002 & .100000000000000000+001 \\ .140000000000000000+002 & .100000000000000000+001 \\ .145000000000000000+002 & .100000000000000000+001 \\ .150000000000000000+002 & .100000000000000000+001 \\ .155000000000000000+002 & .100000000000000000+001 \\ .160000000000000000+002 & .100000000000000000+001 \\ .165000000000000000+002 & .100000000000000000+001 \\ .170000000000000000+002 & .100000000000000000+001 \\ .175000000000000000+002 & .100000000000000000+001 \\ .180000000000000000+002 & .100000000000000000+001 \\ .185000000000000000+002 & .100000000000000000+001 \\ .190000000000000000+002 & .100000000000000000+001 \\ .195000000000000000+002 & .100000000000000000+001 \\ .200000000000000000+002 & .100000000000000000+001 \\ .205000000000000000+002 & .100000000000000000+001 \\ .210000000000000000+002 & .100000000000000000+001 \\ .215000000000000000+002 & .100000000000000000+001 \\ .220000000000000000+002 & .100000000000000000+001 \\ .225000000000000000+002 & .100000000000000000+001 \\ .230000000000000000+002 & .100000000000000000+001 \\ .235000000000000000+002 & .100000000000000000+001 \\ .240000000000000000+002 & .100000000000000000+001 \\ .245000000000000000+002 & .100000000000000000+001 \\ .250000000000000000+002 & .100000000000000000+001 \\ .255000000000000000+002 & .100000000000000000+001 \\ .260000000000000000+002 & .100000000000000000+001 \\ .264999999999999999+002 & .100000000000000000+001 \\ .270000000000000000+002 & .100000000000000000+001 \\ & \end{array}$

$.376921448565488099-040$ $.208848758376254514-044$ $.703592809017752444-049$ $.144086613794369515-053$ $.179330964357678251-058$ $.135626116920590467-063$ $.623194278197991275-069$ $.173955731546672518-074$ $.294943311325798927-080$ $.303722984775031350-086$ $.189939594197950386-092$ $.721299417245120960-099$ $.166320164004887325-105$ $.232848575157153193-112$ $.197913057555326932-119$ $.102122801509426159-126$ $.319886381234348273-134$ $.608236923181640276-142$ $.701996157498568456-150$ $.491772283925648080-158$ $.209095414792273150-166$ $.539586561160790480-175$ $.845084236957246622-184$ $.803245387102246359-193$ $.463333653965844984-202$ $.162190586093347461-211$ $.344534886046460488-221$ $.444126594808806166-231$ $.347405949564997476-241$ $.164898258315193574-251$ $.474936126406738376-262$ $.830017257119653210-273$ $.880166269072796021-284$ $.566319240885615157-295$ $.221090766426373696-306$ .000000000000000000

(Paper 74B3-331) 